\title{
La ética y la crisis de la salud en México
}

\section{Ethics and health crisis in Mexico}

\section{Dra. Marta E. Piña Barba}

Asociación Mexicana Contra el Cáncer Mamario. México, D.F.

\section{RESUMEN}

El objetivo del trabajo es analizar la crisis de la salud en México a partir de un concepto de Ética en la época clásica, surgido de una dicotomía entre las ideas de "vivir correctamente" y "vivir virtuosamente". Se demuestra que esta dicotomía ha continuado y se ha profundizado en el sistema capitalista, y que a su vez ha adquirido la lógica del capital inherente a este período histórico de globalización neoliberal, cuestión esta no solo inherente a México, sino a otros países de la Región y del mundo.

Palabras clave: ética, salud, sistema, globalización neoliberal.

\begin{abstract}
The objective of this paper is to analyze the health crisis in Mexico, taking as a basis the concept of Ethics of the classical times, which stemmed from a dichotomy between the ideas of "living properly" and "living with virtues". It is proved that this dichotomy continues and goes deeper in the capitalist system. At the same time, it acquires the logics of the capital inherent to this historical period of neoliberal globalization, a situation that is not only found in Mexico but also in other countries of Las Americas and in the world as well.
\end{abstract}

Key words: ethics, health, system, neoliberal globalization. 


\section{NTRODUCCI ÓN}

México es un país de una gran extensión territorial, con aproximadamente 113 millones de habitantes, que posee grandes recursos naturales, pero que sin embargo, al igual que otros países de la Región, la salud se encuentra en una grave crisis.

El análisis de la Crisis de la Salud en México la realizo a partir de un concepto de Ética en la época clásica, surgido de una dicotomía entre las ideas de "vivir correctamente" y "vivir virtuosamente" que en primera instancia parecerían ser idénticas, pero no lo son. Se expone pues la idea de que "vivir correctamente" implica vivir según las reglas del sistema, es decir, es una forma de actuar impuesta sistemáticamente, mientras que la segunda "vivir virtuosamente" implica vivir según principios morales que toman en cuenta el bienestar de otros, del "otro". Esta dicotomía ha continuado en las distintas épocas y se ha profundizado en el sistema capitalista adquiriendo la lógica del capital inherente de cada período histórico hasta la globalización neoliberal de este momento.

Para esta aproximación con vistas a entender esta crisis, y para que sirva de acicate a otros investigadores de México y de América Latina con similares situaciones en sus respectivos países, he dividido metodológicamente el trabajo tomando como base la situación de mi país

\section{EL MÉXI CO POSREVOLUCI ONARIO}

Algunos de los principales logros en el México posrevolucionario que se obtuvieron como resultado de la lucha revolucionaria, fue la creación de instituciones de seguridad social y apoyo a la población; desde educación pública, hasta sistemas de salud gratuita que han sido primordiales en la protección del bienestar del pueblo mexicano. La figura de Lázaro Cárdenas del Río, y de otros revolucionarios como I gnacio García Tellez, fueron de trascendental importancia para la instrumentación del nacimiento y desarrollo de instituciones como el Instituto Mexicano del Saguro Social (IMSS), el Instituto de Seguridad Social de los trabajadaores del Estado (ISSTE), Petroleos de México(PEMEX) y otras, el auge en la educación en general y en particular la educación médica, que a su vez se tradujo en resultados reales en incremento en expectativas y calidad de vida en todo el país. Esto en cuanto a la salud se refiere claro está, porque en materia de educación también los logros fueron inmensos y para muestra tenemos la creación del Instituto Politécnico Nacional (IPN), la autonomía universitaria y la modernización de la universidad, la creación de las normales rurales de excelencia, entre otras, pero nuestro tema es la salud. Esta era la época del México posrevolucionario con fuertes influencias socialistas, donde la ética del estado estaba firmemente plantada en lograr la prosperidad de la población.

Desafortunadamente ese período duró poco y no llegó a consolidarse por completo, y con la guerra fría comenzó a su vez el lento desmantelamiento de todas estas instituciones y tendencias de inspiración izquierdista. Hoy en día lo que nos queda de esa época dorada de logros sociales es muy poco y cada vez es menos, pues se obsertva la clara tendencia a su desaparición o más bien, a su aniquilamiento, con la tendencia a desplazar la responsabilidad del estado hacia la iniciativa privada, con la consecuencia lógica de cambio de valores y objetivos.

Esto sin duda ha sido un largo proceso de degradación que ha incluido una transformación incluso de la ética de la salud misma, y que ha ido aparejada con 
los cambios del sistema económico y como consecuencia de dichos cambios; cambios que han venido sucediendo desde ese tiempo posrevolucionario hasta la fecha y que explican la razón por la cual la ética de la salud en México ya no está basada en la búsqueda de ese bienestar general para la población, sino es más bien una ética sistémica basada en la lógica del capitalismo que todo lo transforma en mercancía, la lógica del capital que solo busca maximizar ganancias y todo esto lo hace a costa de restar la seguridad de la población; o sea, corrompe por completo el fin por el cual se crearon esas instituciones en su origen.

Se trata entonces de una ética distinta, la ética del capital que todo lo daña en aras de la máxima plusvalía , y que ha desplazado y está aniquilando la ética social que en algún momento existió en el país, y que lejos de seguir evolucionando a estados superiores de beneficio social, se ha perdido casi por completo.

\section{SITUACIÓN DE LA SALUd EN EL SECTOR PÚBLICO EN MÉXICO}

Existe en el país servicios médicos aparentemente para toda la población, pero eso en realidad es una falacia pues la población ha aumentado en forma no correspondida con la infraestructura hospitalaria y tiene actualmente sobresaturación de pacientes en todas las instituciones existentes, desabastecimiento de material médico, medicamentos y todo tipo de necesidades, para brindar una buena atención médica. El trato médico paciente es pésimo y degradante, debido a la misma sobresaturación abrumadora de trabajo para una cantidad cada vez más reducida de personal, es totalmente impersonal y hasta denigrante, derivado de las pésimas condiciones del entorno general anímico y clínico.

A su vez, encontramos que a todos los niveles existe falta de equipos y de medicamentos, lo que se traduce en pacientes que a pesar de llegar a alcanzar diagnósticos correctos, no logran tener el tratamiento apropiado, sino meramente aquel que se les puede proveer bajo las condiciones mínimas existentes.

Todo lo anterior refleja la lógica de la plusvalía, donde se busca cumplir con la obligación de proveer el servicio social que "justifique" la existencia de la institución o del estado como meta, pero exprimiendo los recursos al máximo como consecuencia de eso.

Esta es una expresión más de la ética capitalista en la salud contemporánea: los sistemas de salud estatales no están ahí para proveer bienestar, sino más bien para justificar la legitimidad del Estado y a su vez, causar el menor drenaje posible de recursos.

\section{SITUACIÓN DE LA SALUd EN EL SECTOR PRI VADO EN MÉXI COํํำ}

Para comprender de forma correcta la manera en la cual la salud se concibe en la época neoliberal, es útil regresar a una época anterior, inclusive al advenimiento del concepto de los Derechos Humanos durante la llustración. Desde ese entonces la idea de que los seres humanos somos iguales y por lo tanto tenemos los mismos derechos, y de que existen derechos fundamentales incluyendo los más básicos como son a la vida y a la salud, son principios incuestionables. Podemos así concluir que a partir de ese momento se concibieron estos derechos humanos como la base de toda ética.

Sin embargo, con los avances del capitalismo, en especial a finales del siglo $20, y$ con el surgimiento del capitalismo neoliberal, la salud se convierte ya no en un 
derecho humano básico, sino en un bien a comercializar; y es así que se descubre a la salud como una industria, que es tal vez una de las más ricas que existen pues nos vende algo que absolutamente todos los seres humanos necesitamos. Así es como de pronto, el hecho de que todos los seres humanos seamos iguales significa más bien que todos somos potenciales consumidores del mismo producto, y de toda la parafernalia necesaria para obtenerlo en su forma ideal.

Y hablo de forma ideal pues en cuanto surge esta diferenciación, también emerge una distinción de consumidores. Como con cualquier otro bien a la venta, la población ya no adquiere la salud que necesita, sino la salud que tiene capacidad de adquirir a la compra. Y en este espacio se crea un espectro muy amplio: desde los sectores más pobres que solo son capaces de adquirir los tratamientos básicos, hasta los sectores económicamente pudientes, los que inclusive adquieren lo que podemos llamar salud de élite, que sin duda redefine el concepto de necesidad.

Este punto es crítico para entender la salud en el neoliberalismo: la desaparición del concepto de necesidad. Basado en una ética sistémica capitalista, la salud como necesidad deja de existir. Solo existe un producto a vender al mejor postor. Los sectores de bajos recursos, al no tener capacidad adquisitiva, no son necesarios para el sistema, y por lo tanto se les puede ofrecer una salud mínima, ya que su supervivencia o calidad de vida no entra en la ecuación. Por el otro lado, a los sectores de alta capacidad adquisitiva se les puede redefinir el concepto de salud a incluir todo tipo de nuevos conceptos, desde estéticos, hasta nuevas enfermedades, "sensaciones de bienestar" que van mucho más allá de cualquier concepto de necesidad.

Ante esta perspectiva no sorprende que tanto empresas transnacionales como el gran capital privado vean en esta "industria" una oportunidad de negocio inmejorable. A final de cuentas, qué puede ser mejor que venderle a la gente un producto intangible, inclusive invisible, pero que todos "necesitamos". Surge entonces toda una cadena de producción creada para obtener mayores ganancias de la venta de la salud.

En primer lugar, los hospitales privados comienzan a operar con una lógica similar al de la hotelería, en la que en lugar de buscar la sanación del paciente, se intenta que el mismo consuma lo más posible durante su estancia, a pesar de ser esta involuntaria. Se reemplazan materiales fácilmente esterilizables por materiales desechables, se adquieren equipos ultraavanzados cuya utilidad es muchas veces cuestionable (al menos considerando el costo abrumador que significan para el paciente), y se les impone una serie de comodidades que no son más que costos agregados y mayores plusvalías, que sin duda no repercute en nada o poco en la curación del enfermo.

Una segunda etapa la representan las industrias farmacéuticas internacionales que, en su afán por obtener las mayores ganancias posibles de sus productos, se esfuerzan con todo tipo de artimañas legales, en limitar la libre circulación de sus productos, por lo que pueden venderlos a precios estratosféricos. El resultado es que inclusive se crean distinciones de clase basadas en el tipo de medicamentos que alguien puede adquirir, y los de uso más crítico son económicamente prohibitivos para la población en general. No es lo mismo ser de la clase social que puede consumir medicamentos de marca, que quienes consumen genéricos, y peor aún, similares. Algunos no tienen acceso ni a estos, pues simplemente no existen para el mercado, y por ende para el sistema. A su vez, cabe mencionar que en el proceso de investigación de estas empresas, es común tanto la invención de nuevas enfermedades, que a su vez crean una demanda por nuevos productos farmacéuticos, como el hecho de que tales productos tengan que ser probados en 
poblaciones humanas, y que las de nuestros países sean de las favoritas para esto dadas las regulaciones excesivamente laxas de las autoridades locales. En este aspecto la salud "pública" tiene la población adecuada para que se experimente en ella precisamente..., dejando los resultados ya al alcance de las poblaciones económicamente más privilegiadas.

Un tercer paso en la cadena de privatización de la salud lo cubren los Seguros de Gastos Médicos, que venden un paso más allá en la alienación de la salud: ellos ya no venden servicios médicos, sino la posibilidad de acceder a la salud en caso de necesitarla. Sin embargo, bajo la misma lógica de la plusvalía, y de la ética de obtener el mayor beneficio al menor costo, sus operaciones se basan en pagar lo menos posible en casos de ser necesitados, a la vez que a la más mínima excusa suben los costos de sus tasas y otros y se valen de cualquier artimaña para lograr su objetivo principal que es siempre su propia plusvalía y nunca la salud del paciente.

Esto nos lleva a la última etapa de la práctica médica privada en el neoliberalismo: la relación médico paciente. Considerando que el médico es el intermediario natural entre el paciente y las industrias médicas antes descritas, es él mismo quien se convierte en el eje de la industria en su totalidad. En otras palabras, es el médico quien se convierte en el vendedor directo del producto salud que estas industrias ofrecen de forma tan ventajosa. Por lo mismo, es el médico quien se encuentra en el centro del dilema moral de qué productos ofrecer y cómo a los potenciales compradores. Pero no simplifiquemos el asunto, pues para el mismo médico no es una decisión simplemente moral sino también de supervivencia en donde se le coloca entre la espada y la pared y generalmente ni siquiera se percata del asunto debido a que el grado de alienación de la mayoría de los galenos ya a estas alturas es total , y ahí salta otra vez más la ideología del sistema capitalista neoliberal en un círculo vicioso de causa efecto.

Considerando que la práctica de la medicina social, que era común y hasta bien remunerada hace apenas unas décadas, hoy en día provee salarios verdaderamente miserables, los médicos están obligados a refugiarse en la medicina privada para poder obtener una subsistencia apropiada. Pero como las reglas económicas del juego en ese espacio las escriben las industrias, el médico acaba teniendo que negociar entre su juramento hipocrático, y la posición de vendedor de pócimas neoliberales.

\section{EL CUERPO EN EL NEOLI BERALI SMO²}

Como habrá quedado ya claro anteriormente, una de las grandes estrategias para poder comercializar la salud en el capitalismo avanzado es su redefinición. Retomando a Marx, e inclusive a Darwin, somos lo que hacemos para subsistir. En ese sentido, no debería existir relación más orgánica o natural que entre nosotros y nuestros cuerpos. La materialidad que nos permite producir y reproducirnos es la esencia básica de nuestra identidad, y sin embargo no es así. Creemos en existencias más allá del cuerpo que nos definen trascendentemente, y que nos inoculan de las dificultades y limitaciones del mismo.

Pero estas definiciones no surgen de nuestras experiencias personales, ni siquiera de las que compartimos con otros en el proceso de socialización. Estas definiciones nos son impuestas por un sistema que necesita equiparar al cuerpo humano con una maquinaria industrial, para así poder someterlo a las mismas leyes económicas con las que rige el resto de su lógica. 
Es de esta forma que en el momento en que una mujer se embaraza su cuerpo deja a ser de ella para pasar al control del aparato médico, y por lo mismo, el bebé deja de ser un humano en potencia para convertirse en "el producto."

Es así como la lógica del capitalismo se va inyectando a lo más íntimo de nuestro ser, nuestro cuerpo, y al reescribirlo, reescribe también nuestra identidad.

Perdemos la unidad natural con nuestro cuerpo para convertirnos en sujetos ajenos a esta maquinaria carnosa. Sujetos que no solo no la controlan, sino que somos víctimas de sus deficiencias, sean estas impulsos, instintos, fallas, deterioro, y eventualmente su muerte (que por esta trágica unión se convierte en la nuestra).

Mi cuerpo y yo somos otros, somos distintos. Es de esta forma que el cuerpo entra en venta, y puede ser analizado, subdividido y comercializado. Se crean nuevos mercados donde se comercializan los instintos sexuales, pero también las dolencias, e inclusive su forma estética.

Pero el estar atrapado dentro de esta maquinaria no solo significa que estamos sujetos a la lógica del mercado para lograr nuestra subsistencia, sino que estamos ante la constante amenaza de que, como cualquier otro aparato de nuestra propiedad, se descomponga sin que entendamos el porqué.

Palabras como infarto, tumor, derrame, son signos misteriosos que nos recuerdan que esta máquina, más que mantenernos con vida, puede fallar en cualquier momento y acabarnos. Caminamos dentro de una bomba de tiempo. La diferencia entre este futuro incierto y aterrador, y la estabilidad, aunque sea momentánea, es uno de los bienes más preciados: la salud.

Pero si la salud es entre otras cosas también la ausencia de enfermedad, es decir, estamos sanos cuando nuestro cuerpo funciona correctamente, y como no entendemos lo que significa el correcto funcionamiento del cuerpo, entonces la salud nos es un signo igualmente indescifrable.

Es esta incertidumbre que nos lleva a adquirir "la salud" en cualquiera que sea el paquete en que nos sea vendida. Ya sea un yogurt con bífidos, un cereal con fibra extra, una pastilla que reduce el colesterol, un anillo que nos limpia el aura, un alineador de chacras, o las flores de Bach, la salud es algo que sabemos que necesitamos, aun si no sabemos cómo funciona. Es el mítico tónico, la piedra filosofal, el grial sagrado, es ese bien que, de ser nuestro dará sentido a toda nuestra existencia. Es por ello que la sabiduría popular nos dice que si tienes salud, todo lo demás es secundario.

Es así como caemos en una de las grandes ironías de esta lógica perversa, vendemos nuestro cuerpo y su labor para obtener, principalmente, algo que nos asegure que este mismo cuerpo siga funcionando.

¿Pero quién es ese gran dador al que le vendemos nuestro caparazón fibroso a cambio de unos momentos más de funcionamiento? Es el mercado todopoderoso. Es a este Dios a quien rendimos tributo para que nos regale unos días o años más con los cuales producirle aún más riquezas. En otro revés irónico, también aquellos que obtienen plusvalía al de vender el producto salud, lo hacen también para alimentar las necesidades y urgencias de sus propios cuerpos descontrolados, es decir enfermos.

El único que no sufre de enfermedades es el mercado. 


\section{LA ÉTI CA EN EL CAPITALISMO AVANZADO}

Para que un tumor crezca o se malignice se requiere que una serie de mutaciones sucedan al interior de la célula. Lo mismo podemos decir a nivel social en términos marxistas: "dentro de los principios de la dialéctica, todo sistema acaba encontrando su fin por la reproducción de sus contradicciones internas."

En este sentido la ética es como el ADN. Es la base recombinante sobre la cual la lógica de todo el sistema se construye. Es la fuente básica de legitimidad. Sin embargo, en el caso de la salud observamos que el capitalismo lleva a una ética que se voltea contra sí misma como una mutación contra la propia célula.

La ética capitalista en la salud, que privilegia las ganancias por encima del bienestar social, y que por lo mismo acaba extrayendo las últimas gotas de plusvalía a cambio de la calidad de vida y supervivencia de la población, se acaba convirtiendo en un arma pasiva de destrucción masiva.

Con la polarización social derivada del capitalismo en esta etapa, y más aún a escala mundial, que es el complemento natural de la globalización neoliberal, cada vez son menos los que tienen acceso a la Salud con S mayúscula, y más los que simplemente no pueden más que observarla como un bien inalcanzable. Este mismo proceso lleva a una sociedad donde la enfermedad y la muerte se conviertan en situaciones cada vez más cotidianas, a pesar de los avances tecnológicos y científicos que supuestamente la sociedad ha desarrollado justamente para evitarlas. I rónicamente, al acabar exprimiendo las ganancias en el proceso de la salud, el sistema capitalista acaba literalmente matando de enfermedad a sus potenciales consumidores, los pacientes, y de hambre a sus trabajadores, los médicos, y entonces lentamente se sofoca a sí mismo.

Ante este catastrófico panorama actual se levanta como un faro en el horizonte la ética socialista, antagónica a la ética del gran capital y cuyo reconocimiento del derecho del "otro" nos vuelve a colocar en un plano de vida y no de muerte y con esto quizás podremos salvar al ser humano de la catástrofe que lo lleve a su desaparición sobre este planeta o incluso a la desaparición del planeta mismo.

Edward Said, ${ }^{3}$ describe que en los procesos imperialistas la categoría del "otro" se crea como una forma de crear un reflejo negativo con el cual justificar la colonización. Pero si entendemos que el otro no es un ser exótico, salvaje, raro, extraño, a quién debemos normalizar o inclusive "curar" sino simplemente un ser humano expresando su diversidad cultural, debemos entender que es nuestra obligación el defender su derecho a vivir de manera saludable, es decir, con el mayor bienestar posible según su propia definición.

En ese sentido es útil recordar la idea del hombre nuevo del Che Guevara:

Mientras existamos dentro de las condiciones objetivas y productivas del capitalismo, estaremos atados a su ética, y a su lógica destructiva. En cuanto logremos ir transformando las condiciones que nos impone el sistema, estas irán alterando nuestras condiciones subjetivas, nuestra moral, hasta que la idea misma de una ética capitalista, de una salud capitalista, parezca un arcaicismo absurdo. ${ }^{4}$ 
El deterioro del ecosistema mundial es evidente, al igual que el deterioro de la "ética", de la moral en este sistema capitalista neoliberal que vivimos y que ya repercute de manera seria y muy peligrosa en la impartición de la salud en la comunidad humana.

El empeoramiento de los principales indicadores de salud, el deterioro del nivel de vida, el acortamiento de la esperanza de vida promedio, el aumento de las enfermedades y muertes evitables, en particular en la infancia; la profunda desigualdad en la atención de la salud en las distintas clases sociales, la corrupción moral galopante de los galenos y demás trabajadores se la salud, la potencial capacidad de destrucción del ser humano, todo esto y más, constituye una muestra abigarrada de indiferencia, desidia, desigualdad, injusticia, miseria y muerte, que inexorablemente acompaña a la política neoliberal y que abarca sin duda alguna a "la salud" de tal forma que solo tiene derecho a tener salud y a poder vivir sanamente el que tiene suficiente dinero para costearla, como mercancía, el resto de la población se puede morir sin que esto a nadie le importe ni le incomode... ...Esta es la "ética" de la salud en este capitalismo neoliberal que vivimos.

\section{REFERENCI AS BI BLI OGRÁFICAS}

1. Piña Barba M. Situación de comercialización de la Salud en el Sector Privado. México: ENAH; 2008.

2. Madrazo I. El Sujeto en la Salud en el Capitalismo Avanzado. México: ENAH; 2008.

3. Said E. Culture and Imperialism. New York: Vintage Books; 1993.

4. Cormier J. La Vida del Che. Buenos Aires: Ed. Sudamericana; 1997.

Recibido: 27 de noviembre de 2011.

Aprobado: 25 de enero de 2012.

Marta E. Piña Barba. Hospital Ángeles del Pedregal. Camino a Sta. Teresa 1055815. Col. Héroes de Padierna, Magdalena Contreras. México, D.F. Teléf.: (55)55688091, (55)5568-9151. Correo electrónico: martapina@amecma.org 